\title{
Voluntary cognitive screening: characteristics of participants in an Asian setting
}

This article was published in the following Dove Press journal:

Clinical Interventions in Aging

20 April 2015

Number of times this article has been viewed

\author{
Vanda $\mathrm{Ho}^{1,2}$ \\ Nur Hani Zainal' \\ Linda Lim' \\ Aloysius $\mathrm{Ng}^{\prime}$ \\ Eveline Silva' \\ Nagaendran Kandiah ${ }^{1,3}$ \\ 'Department of Neurology, National \\ Neuroscience Institute, Singapore; \\ ${ }^{2}$ School of Clinical Medicine, \\ University of Cambridge, Cambridge, \\ UK; ${ }^{3}$ Duke-NUS Graduate Medical \\ School, Singapore
}

\begin{abstract}
Background: Mild cognitive impairment (MCI) and dementia are reaching epidemic proportions in Asia. Lack of awareness and late presentation are major obstacles to early diagnosis and timely intervention. Cognitive screening may be an effective method for early detection of dementia in Asia. The purpose of this work was to study the characteristics of subjects volunteering for cognitive screening in an Asian setting and to determine the prevalence of MCI.
\end{abstract}

Methods: Retrospective and cross-sectional data from community subjects attending a screening program from 2008 to 2013 were analyzed. Information on demographics, vascular risk factors, subjective symptoms, and cognitive measures were analyzed over the 6-year period.

Results: Over the 6 years from 2008 to 2013, 1,243 community subjects voluntarily turned up for cognitive screening (91.2\% were Chinese, 5.23\% were Indian, $1.37 \%$ were Malay, and $2.25 \%$ were Eurasian). The mean age of the participants was 61.3 years and the mean number of years of education was 11.0 years. A total of $71.1 \%$ of participants were living in public housing, $59.8 \%$ had at least one cardiovascular risk factor, and 56.2\% reported subjective cognitive symptoms. Over a period of 6 years, no significant change in demographic or clinical variables was noted. High cholesterol and hypertension were consistently the top two risk factors found in the population screened. In total, $17.2 \%$ of the total cohort had MCI. Across the 6 years, the proportion with MCI and depression was relatively constant.

Conclusion: A significant proportion of participants attending voluntary cognitive screening have MCI. Low level of education and presence of vascular risk factors are general predisposing characteristics for MCI, and there are more specific factors pertaining to sex and employment status. Keywords: early detection, screening, cognitive impairment, dementia, vascular risk factors

\section{Introduction}

An estimated 35.6 million people live with dementia worldwide, with a forecast of doubling every 20 years. ${ }^{1,2}$ In Singapore, the overall age-standardized and racestandardized prevalence was $1.26 \%$ based on a study published in $2008 .^{3}$ This lower prevalence compared with other countries in the region may be related to low awareness of dementia in the community and lack of community-based data. It is also likely that only patients with significant dementia were included in the 2008 study by Sahadevan et al and those in the mild stages of dementia were not represented. ${ }^{3}$

Cognitive impairment and dementia has far-reaching social and economic implications. In 2010, the global societal cost of dementia was US\$ 604 billion. ${ }^{1}$ Further, dementia is the main reason for institutionalization in the elderly ${ }^{4}$ and is responsible for driving many families below the poverty line. ${ }^{1,4}$ Thus, informal care is indispensable. ${ }^{5}$ Support for these caregivers come largely from having an early diagnosis and a stable community infrastructure.

By bringing the diagnostic phase one step earlier and screening community dwellers for mild cognitive impairment (MCI), there are significant benefits. MCI is a high-risk
Correspondence: Nagaendran Kandiah Department of Neurology, National Neuroscience Institute, I I Jalan Tan Tock Seng, Singapore 308433

Tel +6563577199

Fax +6563577137

Email nagaendran_kandiah@nni.com.sg 
state for dementia, with annual progression rates to dementia ranging from $12 \%$ to $15 \% .{ }^{6,7}$ Introduction of lifestyle changes such as mentally-stimulating activities and reduction of risk factors may prevent progression. ${ }^{8,9}$ Additionally, alerting patients early when they have most of their mental capacities intact allows for autonomy in future planning, allaying fears of uncertainty with regards to medical treatments ${ }^{10}$ and personal affairs. ${ }^{11}$ Therefore, MCI screening is useful in maximizing ability.

Cognitive screening also forms part of the public health agenda of dementia awareness and education. The Value of Knowing study ${ }^{12}$ found dementia to be one of the most feared diseases second only to cancer. Lack of awareness decreases resilience of the family unit, increases financial and legal vulnerability, and impedes construction of a firm social infrastructure. On the other hand, improved understanding helps eliminate stigmatization, prevents social isolation, and circumvents delays to seeking aid. By promoting public education on the warning signs of dementia and modifiable cardiovascular risk factors, it is hoped that the general public can take active measures to reduce their susceptibility to dementia.

International organizations have placed much emphasis on such efforts. The Kyoto Declaration by Alzheimer's Disease International ${ }^{13}$ released minimum recommendations for dementia care, including monitoring community health. To supplement these guidelines, the World Health Organization launched the Mental Health Gap Action Programme, ${ }^{14}$ with dementia as a priority non-communicable condition. In Singapore, the Health Promotion Board's strategic dementia plan ${ }^{15}$ raised national awareness via various modalities, such as mass media and community engagement. Hence, the way forward is identification of reliable and locally relevant estimates of the country's need in order to develop effective national dementia policies.

There has been much research describing risk factors for cognitive impairment, although most have conflicting results. This ambiguity could be attributed to the different unique population groups studied, emphasizing the importance of local data for policy development. Of the more common risk factors examined, education level has consistently been found to be protective. ${ }^{16,17}$ Cardiovascular risk factors such as high serum total cholesterol, ${ }^{16}$ hypertension, ${ }^{17}$ stroke, ${ }^{18}$ and diabetes ${ }^{19}$ have been found to be associated. These parameters are useful in describing the cohort susceptible to cognitive impairment so that steps can be taken to identify and monitor them, and if possible, improve modifiable risk factors.
This is one of the first studies to examine an Asian population who voluntarily take up cognitive screening. This naturalistic approach indirectly informs about the cohort with high awareness. Risk factors for MCI can be evaluated, both in general and for specific groups, and mismatch in characteristics between those screened to be cognitively intact and those with MCI can then be identified. These data can be used to inform awareness-raising campaigns that can be tailored specifically for the more susceptible group in order to maximize effectiveness and target screening.

\section{Materials and methods}

This is a retrospective study of urban, multi-ethnic, communitydwelling participants who voluntarily attended cognitive screening organized by the National Neuroscience Institute in Singapore from 2008 to 2013.

\section{Screening program}

The screening was organized annually and was conducted over a course of 1 day, with different cohorts enrolled into the database registry each year. The screening event was open to all Singapore residents over the age of 50 years with no prior diagnosis of a cognitive disorder. The event was publicized through local mainstream media, including radio programs and print media (eg, flyers, newspaper advertisements), in the main languages, including English, Mandarin, Malay, and Tamil. Participants were required to telephone in advance to make appointments for the cognitive screening. Registration could be made by the participant or their next-of-kin. At the time of the screening event, participants provided informed consent to be entered into the study.

Screening assessments were conducted by trained staff. Raters underwent training sessions prior to the event and were instructed to follow standardized protocols and instruction manuals for each cognitive test to ensure standardized administration. Each participant went through a three-step process:

1. Questionnaire for demographic and clinical data, including type and duration of any cognitive symptoms, use of medications, and comorbidities. They were specifically required to indicate the presence of diabetes mellitus, hypertension, high cholesterol, stroke, heart disease, and history of smoking. For demographic features, a high level of occupation referred to a job position demanding complex skills, planning, judgment, and higher qualification (eg, professional or managerial posts), whereas a low level one referred to a job position demanding simple skills, manual work, and lesser qualifications. 
This includes unemployment, having an unskilled (eg, cleaner) or semi-skilled job (eg, taxi driver). High socioeconomic status required three or more of the following: high level of education, employed, high level of occupation, or private residence.

2. Cognitive screening battery: locally validated 30-point Mini-Mental State Examination (MMSE), 30-point Montreal Cognitive Assessment (MoCA), Frontal Assessment Battery (FAB), 15-point Geriatric Depression Scale (GDS), and 8-point Even Briefer Assessment Scale for Depression. ${ }^{20-23}$ All four questionnaires were forward and back-translated into Mandarin, Tamil, and Malay to cater for the multi-ethnic population in Singapore.

3. One-to-one structured interview on cognitive symptoms using the Memory Assessment Questionnaire, a brief index of memory complaint, ${ }^{24}$ and the Instrumental Activities of Daily Living, ${ }^{25}$ also culturally validated in Singapore. ${ }^{26}$

\section{Diagnostic process}

At the end of the three-step process, a clinical diagnosis of MCI, dementia, or no cognitive impairment was made. MCI was diagnosed based on the Petersen criteria, ${ }^{27}$ whereby: the participants and/or informant reported objective cognitive decline for at least the previous 6 months; scores on global cognition were 1.5 standard deviations below the locally validated norms for age and education; ${ }^{28}$ functional abilities of participant were intact; and the participant had no dementia. Dementia was diagnosed based on the Diagnostic and Statistical Manual of Mental Disorders, Fourth Edition-Text Revised (DSM IV-TR) criteria. ${ }^{29}$ Depression was diagnosed in participants with a Geriatric Depression Scale score $>4$ or Even Briefer Assessment Scale for Depression score $>2{ }^{23}$

\section{Statistical analysis}

Analyses were carried out using Stata version 10.2 software (Statacorp, College Station, TX, USA). For descriptive analyses, Mann-Whitney $U$-tests for continuous variables and chisquare tests for categorical variables were used to compare means for the demographic, clinical, and cognitive variables. Fisher's exact test was used for nominal variables if the cell count reached $\leq 5$. Mann-Whitney $U$-tests were utilized as the data were non-parametric based on Shapiro-Wilk tests (all $P<0.05$ ). Sociodemographic, risk factor, and cognitive characteristics were described as a cohort, separately by year and by presence of cognitive impairment. A series of omnibus Kruskal-Wallis tests was used for comparison of variables across the years. Comparison between younger ( $\leq 60$ years) and older ( $\geq 61$ years) subjects was performed using a median split. Multivariate logistic regression analyses adjusting for age, education, ethnicity, sex, and employment were carried out. The level of statistical significance was set to $P \leq 0.05$.

\section{Results}

Over the 6 years from 2008 to 2013, 1,243 community participants $(67.0 \%$ female, $33 \%$ male) voluntarily turned up for cognitive screening. In total, $91.2 \%$ were Chinese, $5.2 \%$ were Indian, 1.3\% were Malay, and 2.2\% were Eurasian. The mean age of the participants was $61.3 \pm 8.08$ years. The mean number of years of education was $11.0 \pm 3.85$, with $87 \%$ having secondary education or higher. In total, $71.1 \%$ of participants were living in public housing, 39.2\% were employed, and $55.7 \%$ had or were holding high level jobs (Table 1). In terms of risk factor profile, 59.8\% had at least one cardiovascular risk factor; $12.4 \%$ had diabetes mellitus, $35.7 \%$ had hypertension, $39.7 \%$ had high cholesterol, $6.6 \%$ had heart disease, $1.2 \%$ had stroke, and $4.2 \%$ were smokers; $56.2 \%$ reported subjective cognitive symptoms. The

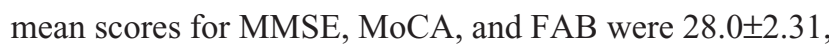
25.9 \pm 2.99 , and 16.4 \pm 1.48 , respectively.

When demographic characteristics were compared over 6 years of cognitive screening, participants were consistently predominantly female and Chinese, which reflects the demographic composition of the country. Most had secondary education or higher, and were currently unemployed (Table 2). Except for the years 2008 and 2013, there had been more participants of high than low socioeconomic status. This finding was similarly reflected in the type of housing, as most lived in public housing. The only year in which more participants held a low level occupation was 2008. For risk factor profile, the majority had at least one cardiovascular risk factor, and this was seen for all years. High cholesterol and hypertension were consistently the top two risk factors found in the population screened, and stroke was the lowest. Although the percentage reporting subjective cognitive symptoms fluctuated across the years, MMSE, MoCA, and FAB scores were relatively constant and the proportion diagnosed to have MCI was similarly consistent. Only a minority were depressed.

From Table 3, 17.2\% of the cohort had MCI. Comparison between no cognitive impairment and MCI populations reveal participants with MCI to be older, of ethnic minority, and to have fewer years of education, even after adjusting for covariates of age, years of education, ethnicity, socioeconomic status, and occupation. History of prior stroke $(1.2 \%$ versus $1.4 \%, P=0.01842$ ) was found to be higher among 
Table I Demographic, risk factor, and cognitive profiles of all subjects

\begin{tabular}{|c|c|}
\hline & Whole cohort $(n=I, 243)$ \\
\hline \multicolumn{2}{|l|}{ Demographic profile } \\
\hline Age, years (mean $\pm S D$ ) & $61.3 \pm 8.08$ \\
\hline \multicolumn{2}{|l|}{ Sex, n (\%) } \\
\hline Male & $410(33.0)$ \\
\hline Female & $833(67.0)$ \\
\hline \multicolumn{2}{|l|}{ Ethnicity, n (\%) } \\
\hline Chinese & I, I33 (9I.2) \\
\hline Malay & $17(1.4)$ \\
\hline Indian & $65(5.2)$ \\
\hline Eurasian & $28(2.2)$ \\
\hline Years of education, years (mean $\pm S D$ ) & $11.0 \pm 3.85$ \\
\hline \multicolumn{2}{|l|}{ Level of education, n (\%) } \\
\hline No formal education & $38(3.06)$ \\
\hline Primary & $125(10.0)$ \\
\hline Secondary & $534(43.0)$ \\
\hline Tertiary & $546(44.0)$ \\
\hline \multicolumn{2}{|l|}{ Housing type, n (\%) } \\
\hline Public & $416(7 \mid . I)$ \\
\hline Private & $169(28.9)$ \\
\hline \multicolumn{2}{|l|}{ Employment, n (\%) } \\
\hline Employed & $487(39.2)$ \\
\hline Unemployed & $756(60.8)$ \\
\hline \multicolumn{2}{|l|}{ Level of occupation, $n(\%)$} \\
\hline High & $597(55.7)$ \\
\hline Low & $475(44.3)$ \\
\hline \multicolumn{2}{|l|}{ Risk factor profile } \\
\hline Diabetes mellitus, n (\%) & $154(12.4)$ \\
\hline Hypertension, n (\%) & $444(35.7)$ \\
\hline High cholesterol, n (\%) & $494(39.7)$ \\
\hline Heart disease, n (\%) & $83(6.7)$ \\
\hline Stroke, n (\%) & $15(1.2)$ \\
\hline Smoking, n (\%) & $46(4.3)$ \\
\hline \multicolumn{2}{|l|}{ Cognitive profile } \\
\hline Subjective cognitive symptoms, n (\%) & $699(56.2)$ \\
\hline MMSE $(n=968)$, mean \pm SD & $28.0 \pm 2.31$ \\
\hline MoCA $(n=I, 072)$, mean $\pm S D$ & $25.9 \pm 2.99$ \\
\hline FAB $(n=704)$, mean \pm SD & $16.4 \pm 1.48$ \\
\hline
\end{tabular}

Abbreviations: FAB, Frontal Assessment Battery; MoCA, Montreal Cognitive Assessment; MMSE, Mini-Mental State Examination; SD, standard deviation.

subjects with MCI. The incidence of depression was not found to be different.

Table 4 describes the population stratified based on age. Participants aged $\geq 61$ years had higher rates of MCI, lower education, and a higher burden of vascular risk factors. A comparison based on sex demonstrated subjective memory difficulty to be correlated with cognitive impairment in both females (adjusted odds ratio [OR] 2.60; confidence interval [CI] 1.49-4.53; $P=0.001)$ and males (adjusted OR 7.90; CI 3.17-19.69; $P=0.00001$ ), and specifically for females, education (adjusted OR 0.27; CI 0.10$0.76 ; P=0.013$ ) and high cholesterol (adjusted OR 0.51;
CI $0.28-0.93 ; P=0.03)$ were implicated. When analyses were performed based on employment status, age was significantly associated with MCI among employed subjects (adjusted OR 1.08; CI 0.04-0.12; $P=0.00001$ ) while education was significantly associated with MCI among the unemployed (adjusted OR 0.09; CI $-4.53-0.31 ; P=0.00001$ ).

\section{Discussion}

In this study, we report on a voluntary cognitive screening Asian population across 6 years. Our findings demonstrate that females and those with higher education, higher socioeconomic status, and pre-existing vascular risk factors are more likely to attend for cognitive screening voluntarily. A significant proportion (17.2\%) of community-dwelling subjects who attended screening was found to have MCI. Participants with MCI were older, female, and had fewer years of education.

When compared with other screened groups, the characteristics of this population attending for cognitive screening in Asia closely resemble those of their western counterparts. Previous studies conducted in the USA have looked at screened populations as well. Lawrence et al targeted a more elderly population. ${ }^{30}$ Aside from the older age, our results are consistent with their predominance of females and more educated subjects. Another voluntary study using data from the free Memory Screening Outreach Program ${ }^{31}$ also has a similar demographic profile, and found depression to be present in only a minority (17.0\%). The cohort with a higher probability of cognitive impairment in that program was older and had fewer years of education, which correlates well with our MCI population.

The majority of participants reported subjective cognitive symptoms but were not depressed, and this might be their primary reason for attending voluntary screening. Further, of those who reported such symptoms, a significant proportion turned out to have MCI. This finding suggests that people who volunteer for cognitive screening have genuine cognitive deficits which they are aware of. It may be that this group of highly educated people are more sensitive to changes in their cognitive function, perhaps due to intellectually demanding jobs or hobbies. Notably, while many of them reported subjective cognitive symptoms, they did not present to their doctors. This reluctance to seek professional help may be attributed to social stigma related to cognitive impairment, lack of access to specialized services such as memory clinics, or unwillingness to pay for a seemingly trivial issue. Screening may therefore potentially be helpful in removing these barriers, ${ }^{32}$ in tandem with education to emphasize differences between normal aging and pathological cognitive impairment. 
Table 2 Demographic, risk factor, and cognitive profiles of subjects by year

\begin{tabular}{|c|c|c|c|c|c|c|c|}
\hline & $\begin{array}{l}2008 \\
(n=342)\end{array}$ & $\begin{array}{l}2009 \\
(n=119)\end{array}$ & $\begin{array}{l}2010 \\
(n=93)\end{array}$ & $\begin{array}{l}2011 \\
(n=171)\end{array}$ & $\begin{array}{l}2012 \\
(n=275)\end{array}$ & $\begin{array}{l}2013 \\
(n=243)\end{array}$ & $P$-value \\
\hline \multicolumn{8}{|l|}{ Demographic profile } \\
\hline Age, years (mean $\pm S D)$ & $59.2 \pm 9.02$ & $60.9 \pm 7.22$ & $58.8 \pm 5.63$ & $62.7 \pm 6.44$ & $61.7 \pm 8.27$ & $63.9 \pm 7.66$ & $<0.001$ \\
\hline Sex, n (\%) & & & & & & & $\begin{array}{l}0.0253 \\
\text { (with ties) }\end{array}$ \\
\hline Male & $132(38.6)$ & $33(27.7)$ & $35(37.6)$ & $52(30.4)$ & $94(34.2)$ & $64(26.3)$ & \\
\hline Female & $210(61.4)$ & $86(72.3)$ & $58(62.4)$ & $119(69.6)$ & $181(65.8)$ & $179(73.7)$ & \\
\hline Ethnicity, n (\%) & & & & & & & $\begin{array}{l}0.0204 \\
\text { (with ties) }\end{array}$ \\
\hline Chinese & $304(88.9)$ & $112(94.2)$ & $80(86.0)$ & 157 (91.8) & $247(89.8)$ & $233(95.9)$ & \\
\hline Malay & 0 & 0 & $2(2.2)$ & $\mathrm{I}(0.6)$ & I I (4.0) & $3(1.2)$ & \\
\hline Indian & $34(9.9)$ & $4(3.4)$ & $10(10.8)$ & $5(2.9)$ & $9(3.3)$ & $3(1.2)$ & \\
\hline Eurasian & $4(1.2)$ & $3(2.5)$ & $I(I . I)$ & $8(4.7)$ & $8(2.9)$ & $4(1.6)$ & \\
\hline $\begin{array}{l}\text { Years of education, } \\
\text { years (mean } \pm S D \text { ) }\end{array}$ & $10.0 \pm 3.74$ & $10.9 \pm 3.40$ & $11.6 \pm 3.21$ & $11.9 \pm 3.56$ & $11.6 \pm 4.49$ & $11.0 \pm 3.57$ & $<0.01$ \\
\hline Level of education, $n(\%)$ & & & & & & & $<0.01$ \\
\hline No formal education & $15(4.4)$ & $\mathrm{I}(0.8)$ & 0 & $4(2.3)$ & $6(2.2)$ & $12(4.9)$ & \\
\hline Primary & $67(19.6)$ & $13(10.9)$ & $7(7.5)$ & $8(4.7)$ & $21(7.6)$ & $9(3.7)$ & \\
\hline Secondary & $\mid 43(4 \mid .8)$ & 57 (47.9) & $37(39.8)$ & $62(36.3)$ & $101(36.7)$ & $134(5.5)$ & \\
\hline Tertiary & $117(34.2)$ & $48(40.3)$ & $49(52.7)$ & $97(56.7)$ & $147(53.5)$ & $88(36.2)$ & \\
\hline SES, n (\%) & & & & & & & $<0.01$ \\
\hline High & $100(29.2)$ & $116(97.5)$ & $86(92.5)$ & |4| (82.5) & $244(88.7)$ & $73(30.0)$ & \\
\hline Low & $242(70.8)$ & $3(2.5)$ & $7(7.5)$ & $30(7.5)$ & $31(11.3)$ & $170(70.0)$ & \\
\hline \multicolumn{8}{|l|}{ Housing type ( $n=585), n(\%)$} \\
\hline Public & $254(74.3)$ & & & & & $162(66.7)$ & \\
\hline Private & $88(25.7)$ & & & & & $81(33.3)$ & \\
\hline Employment, n (\%) & & & & & & & 0.0154 \\
\hline Employed & $158(46.2)$ & $38(31.9)$ & $38(40.9)$ & $55(32.2)$ & $126(45.8)$ & $85(35.0)$ & \\
\hline Unemployed & $184(53.8)$ & $81(68.1)$ & $55(59.1)$ & $116(67.8)$ & $149(54.2)$ & $158(65.0)$ & \\
\hline Level of occupation $(n=1,072), n(\%)$ & & & & & & & $<0.01$ \\
\hline High & $36(10.5)$ & $100(84.0)$ & $77(82.8)$ & & $209(76.0)$ & $175(72.0)$ & \\
\hline Low & $306(89.5)$ & $19(16.0)$ & $16(17.2)$ & & $66(24.0)$ & $68(28.0)$ & \\
\hline \multicolumn{8}{|l|}{ Risk factor profile } \\
\hline Diabetes mellitus, n (\%) & $50(14.6)$ & $14(\mid 1.8)$ & $10(10.8)$ & $19(11.1)$ & $37(13.5)$ & $24(9.9)$ & $0.94 I$ \\
\hline Hypertension, n (\%) & $118(34.5)$ & $43(36.1)$ & $26(28.0)$ & $65(38.0)$ & $98(35.6)$ & $94(38.7)$ & 0.739 \\
\hline High cholesterol, n (\%) & $132(38.6)$ & $55(46.2)$ & $29(31.2)$ & $74(43.3)$ & $107(38.9)$ & $97(39.9)$ & 0.497 \\
\hline Heart disease, $n(\%)$ & $26(7.6)$ & $8(6.7)$ & $4(4.3)$ & II (6.4) & $17(6.2)$ & $17(7.0)$ & 0.998 \\
\hline Stroke, n (\%) & $5(1.5)$ & $2(1.7)$ & $\mathrm{I}(\mathrm{I} . \mathrm{I})$ & $2(1.2)$ & $3(1.1)$ & $2(0.8)$ & 1 \\
\hline Smoking $(n=I, 072), n(\%)$ & $27(7.9)$ & $8(6.7)$ & $4(4.3)$ & & $4(1.5)$ & $3(1.2)$ & $\begin{array}{l}<0.0 \text { I } \\
\text { (with ties) }\end{array}$ \\
\hline \multicolumn{8}{|l|}{ Cognitive profile } \\
\hline $\begin{array}{l}\text { Subjective cognitive } \\
\text { symptoms, } \mathrm{n}(\%)\end{array}$ & I54 (45.0) & $70(58.8)$ & $76(81.7)$ & I33 (77.8) & $122(44.4)$ & I 44 (59.3) & $<0.01$ \\
\hline MMSE $(n=968)$, mean \pm SD & $27.6 \pm 2.84$ & $28.7 \pm 2.04$ & $28.6 \pm 0.247$ & $28.2 \pm 2.88$ & & $27.9 \pm 2.30$ & $<0.01$ \\
\hline MoCA $(n=I, 072)$, mean \pm SD & $24.0 \pm 2.47$ & $27.6 \pm 3.11$ & $27.3 \pm 3.44$ & & $26.5 \pm 3.30$ & $26.5 \pm 2.78$ & $<0.01$ \\
\hline $\mathrm{FAB}(\mathrm{n}=704)$, mean $\pm S D$ & $16.3 \pm 2.22$ & $16.8 \pm 0.133$ & & & & $16.4 \pm 1.77$ & 0.0749 \\
\hline Mild cognitive impairment, $n(\%)$ & $129(37.7)$ & $9(7.9)$ & II (II.8) & $14(8.3)$ & $27(9.8)$ & $24(9.9)$ & $<0.01$ \\
\hline Depression (n=797), n (\%) & $65(19.0)$ & $21(18.5)$ & $10(10.8)$ & & & $47(19.3)$ & 0.637 \\
\hline
\end{tabular}

Notes: MMSE and MoCA range 0-30; FAB range 0-18.

Abbreviations: FAB, Frontal Assessment Battery; MoCA, Montreal Cognitive Assessment; MMSE, Mini-Mental State Examination; SD, standard deviation; SES, socioeconomic status.

Those who were cognitively impaired were also more likely to have a low level of education. Cognitive protection conferred by a high level of education is well documented, and is attributed to having a higher cognitive reserve..$^{33,34}$ In addition, minority ethnic status as a predictor for MCI is consistent with findings from prior studies in the Western literature. ${ }^{35}$ Notably, a protective effect of education was found to be more important in those who were unemployed, and this could be attributed to participation in more cognitively interactive hobbies after 
Table 3 Normal cognition versus mild cognitive impairment

\begin{tabular}{|c|c|c|c|c|c|}
\hline & $N C(n=1,029)$ & $\mathrm{MCl}(n=2 \mid 4)$ & $P$-value & Adjusted OR (95\% Cl) & $P$-value \\
\hline \multicolumn{6}{|l|}{ Demographic profile } \\
\hline Age, years (mean $\pm S D)$ & $61.00 \pm 7.57$ & $62.8 \pm 10.0$ & 0.0314 & $\mathrm{I} .04(\mathrm{I} .02-1.06)$ & 0.00002 \\
\hline Sex, n (\%) & & & 0.822 & $1.54(1.08-2.19)$ & 0.71017 \\
\hline Male & $338(32.8)$ & $72(33.6)$ & & & \\
\hline Female & $691(67.2)$ & $142(66.4)$ & & & \\
\hline Ethnicity, n (\%) & & & 0.0017 & & \\
\hline Chinese & $950(92.3)$ & $183(85.5)$ & & Reference point & \\
\hline Malay & $15(1.5)$ & $2(0.9)$ & & $2.63(0.89-7.79)$ & 0.08006 \\
\hline Indian & $39(3.8)$ & $26(12.1)$ & & $2.07(1.15-3.73)$ & 0.0015 \\
\hline Eurasian & $25(2.4)$ & $3(1.4)$ & & $0.91(0.30-2.72)$ & 0.86533 \\
\hline Years of education, years (mean $\pm S D$ ) & $11.4 \pm 3.63$ & $9.12 \pm 4.32$ & $<0.01$ & $1.04(1.00-1.09)$ & 0.03666 \\
\hline Level of education, n (\%) & & & $<0.01$ & & \\
\hline No formal education & $21(2.0)$ & $17(7.9)$ & & $0.91(0.28-2.97)$ & 0.87191 \\
\hline Primary & $74(7.2)$ & $51(23.8)$ & & $1.49(0.5 \mathrm{I}-4.36)$ & 0.46456 \\
\hline Secondary & $447(43.4)$ & $87(40.7)$ & & $\mathrm{I} .47(0.50-4.3 \mathrm{I})$ & 0.48670 \\
\hline Tertiary & $487(47.3)$ & $59(27.6)$ & & Reference point & \\
\hline Housing type, n (\%) & $(n=432)$ & $(n=I 53)$ & 0.408 & & \\
\hline Public & $300(69.4)$ & $116(75.8)$ & & $1.01(0.48-2.13)$ & 0.98033 \\
\hline Private & $132(30.6)$ & $37(24.2)$ & & Reference point & \\
\hline SES, n (\%) & & & $<0.01$ & & 0.88626 \\
\hline Low & $346(33.6)$ & $137(64.0)$ & & Reference point & \\
\hline High & $683(66.4)$ & $77(36.0)$ & & $0.98(0.69-1.38)$ & \\
\hline Employment, n (\%) & & & 0.436 & & \\
\hline Employed & $610(59.3)$ & $133(62.1)$ & & Reference point & \\
\hline Unemployed & $419(40.7)$ & $81(37.9)$ & & $1.09(0.76-1.56)$ & 0.64729 \\
\hline Level of occupation, n (\%) & $(n=872)$ & $(n=200)$ & $<0.01$ & & \\
\hline High & $597(68.5)$ & 0 & & Reference point & \\
\hline Low & $275(31.5)$ & $200(100)$ & & I.3। (0.94-I.82) & 0.10577 \\
\hline \multicolumn{6}{|l|}{ Risk factor profile } \\
\hline Diabetes mellitus, n (\%) & $116(1 \mathrm{I} .3)$ & $38(17.8)$ & 0.0088 & $1.16(0.75-1.79)$ & 0.51083 \\
\hline Hypertension, n (\%) & $357(34.8)$ & $87(40.7)$ & 0.0979 & $1.14(0.83-1.57)$ & 0.42791 \\
\hline High cholesterol, n (\%) & $400(38.9)$ & $94(43.9)$ & 0.170 & $0.97(0.7 \mathrm{I}-1.33)$ & 0.865 \\
\hline Heart disease, $n(\%)$ & $64(6.2)$ & $19(8.9)$ & 0.156 & $\mathrm{I} .65(0.97-2.8 \mathrm{I})$ & 0.06483 \\
\hline Stroke, n (\%) & $12(1.2)$ & $3(1.4)^{\prime}$ & 0.774 & $3.57(1.24-10.26)$ & 0.01842 \\
\hline \multirow[t]{2}{*}{ Smoking, n (\%) } & $(n=872)$ & $(n=200)$ & 0.0131 & $1.14(0.83-1.57)$ & 0.42791 \\
\hline & $31(3.6)$ & $15(7.5)$ & & & \\
\hline Cardiovascular risk factors, n (\%) & & & 0.0039 & & 0.91931 \\
\hline 0 & $426(4 \mid .4)$ & $73(34.1)$ & & Reference point & \\
\hline I & $334(32.5)$ & $65(30.4)$ & & $1.02(0.75-1.39)$ & \\
\hline 2 & $177(17.2)$ & $42(19.6)$ & & & \\
\hline 3 & $79(7.7)$ & $29(13.6)$ & & & \\
\hline 4 & $11(1.1)$ & $5(2.3)$ & & & \\
\hline 5 & $\mathrm{I}(0.0 \mathrm{I})$ & 0 & & & \\
\hline 6 & $\mathrm{I}(0.0 \mathrm{I})$ & 0 & & & \\
\hline \multicolumn{6}{|l|}{ Cognitive profile } \\
\hline \multirow[t]{2}{*}{ Depression, n (\%) } & $(n=624)$ & $(n=173)$ & 0.267 & I.II (0.73-I.68) & 0.62634 \\
\hline & $107(17.1)$ & $36(20.8)$ & & & \\
\hline MMSE, mean \pm SD & $28.47(2.12)$ & $25.66(3.45)$ & $<0.001$ & I.44 (I.32-I.57) & 0.00001 \\
\hline MoCA, mean $\pm S D$ & $27.56(2.91)$ & $24.76(4.76)$ & $<0.001$ & $1.20(1.13-1.27)$ & 0.00001 \\
\hline $\mathrm{FAB}$, mean $\pm \mathrm{SD}$ & $16.72(1.56)$ & $15.48(2.95)$ & $<0.001$ & $1.22(1.10-1.36)$ & 0.00014 \\
\hline
\end{tabular}

Notes: In the multivariate logistic regression model, ORs were adjusted for covariates of years of education, ethnicity, socioeconomic status, and occupation.

Abbreviations: FAB, Frontal Assessment Battery; MoCA, Montreal Cognitive Assessment; MMSE, Mini-Mental State Examination; SD, standard deviation; SES, socioeconomic status; $\mathrm{NC}$, normal cognition; $\mathrm{MCl}$, mild cognitive impairment; $\mathrm{OR}$, odds ratio; $\mathrm{Cl}$, confidence interval.

retirement. However, employment status was not found to be a sociodemographic variable predicting MCI, and this may have been related to the relatively high education in this cohort.
Additionally, most participants had at least one cardiovascular risk factor, possibly because this population with health comorbidities are more likely to have increased contact with health care professionals and so are more likely to attend 
Table 4 Comparison of age groups based on median split

\begin{tabular}{|c|c|c|c|c|c|}
\hline $\begin{array}{l}\text { Total }(n=I, 243) \\
\text { Median } 61.00\end{array}$ & $\begin{array}{l}\leq 60 \text { years } \\
(n=608)\end{array}$ & $\begin{array}{l}\geq 61 \text { years } \\
(n=635)\end{array}$ & $P$-value & $\begin{array}{l}\text { Multivariate } \\
\text { OR }(95 \% \mathrm{CI})\end{array}$ & $P$-value \\
\hline \multicolumn{6}{|l|}{ Demographic profile } \\
\hline Age, years (mean $\pm S D)$ & $54.83 \pm 3.85$ & $67.52 \pm 5.89$ & 0.00001 & NA & NA \\
\hline Sex, $n(\%)$ & & & 0.00005 & $1.84(1.43-2.37)$ & 0.00001 \\
\hline Male & $167(27.47)$ & $243(38.27)$ & & & \\
\hline Female & $44 \mid(72.53)$ & $392(61.73)$ & & & \\
\hline Ethnicity, n (\%) & & & 0.09777 & & \\
\hline Chinese & $560(92.11)$ & $573(90.24)$ & & Reference point & \\
\hline Malay & $12(1.97)$ & $5(0.79)$ & & $0.39(0.13-1.14)$ & 0.08418 \\
\hline Indian & $25(4.11)$ & $40(6.30)$ & & $1.52(0.89-2.59)$ & 0.12951 \\
\hline Eurasian & $11(1.81)$ & $17(2.67)$ & & $1.82(0.81-4.08)$ & 0.1499 \\
\hline \multicolumn{5}{|l|}{$($ mean $\pm S D)$} & 0.00001 \\
\hline Level of education, n (\%) & & & 0.14232 & & \\
\hline No formal education & $21(3.45)$ & $17(2.68)$ & & Reference point & \\
\hline Primary & $58(9.54)$ & $67(10.55)$ & & $1.53(0.72-3.27)$ & 0.2734 \\
\hline Secondary & $279(45.89)$ & $255(40.16)$ & & $1.13(0.57-2.26)$ & 0.72892 \\
\hline Tertiary & $250(41.12)$ & $296(46.61)$ & & $1.16(0.57-2.33)$ & 0.67777 \\
\hline Housing type, n (\%) & $(n=345)$ & $(n=240)$ & 0.38680 & & \\
\hline Public & $250(72.46)$ & $166(69.17)$ & & Reference point & \\
\hline Private & $95(27.54)$ & $74(11.65)$ & & $1.06(0.66-1.71)$ & 0.80746 \\
\hline SES, n (\%) & & & $<0.00001$ & & $<0.0000$ I \\
\hline Low & $286(47.04)$ & $197(3 \mid .02)$ & & Reference point & \\
\hline High & $322(52.96)$ & $438(68.98)$ & & $2.21(1.73-2.8 I)$ & \\
\hline Employment, n (\%) & & & $<0.00001$ & & $<0.0000 \mathrm{I}$ \\
\hline Employed & $351(57.73)$ & $136(2 \mid .42)$ & & Reference point & \\
\hline Unemployed & $257(42.27)$ & $499(78.58)$ & & $5.41(4.15-7.05)$ & \\
\hline Level of occupation, n (\%) & & & $<0.00001$ & & 0.00736 \\
\hline High & $359(59.05)$ & $287(45.20)$ & & Reference point & \\
\hline Low & $249(40.95)$ & $358(54.80)$ & & $1.4 \mathrm{I}(1.10-1.80)$ & \\
\hline \multicolumn{6}{|l|}{ Risk factor profile } \\
\hline Diabetes mellitus, n (\%) & $52(8.55)$ & $102(16.06)$ & 0.00006 & $1.96(1.35-2.85)$ & 0.00045 \\
\hline Hypertension, $\mathrm{n}(\%)$ & $156(25.66)$ & $288(45.35)$ & $<0.0000 \mathrm{I}$ & $2.33(1.82-3.00)$ & $<0.0000 \mathrm{I}$ \\
\hline High cholesterol, n (\%) & $210(34.54)$ & $284(44.72)$ & 0.00024 & $1.52(1.20-1.93)$ & 0.00062 \\
\hline Heart disease, $\mathrm{n}(\%)$ & $29(4.77)$ & $54(8.40)$ & 0.00838 & $1.66(1.01-2.72)$ & 0.0457 \\
\hline Stroke, n (\%) & $6(0.99)$ & $9(1.42)$ & 0.48716 & $1.18(0.40-3.5 \mathrm{I})$ & 0.76294 \\
\hline Smoking, n (\%) & $25(4.11)$ & $21(3.31)$ & 0.45227 & $0.83(0.43-1.59)$ & 0.57118 \\
\hline Cardiovascular risk factors, $\mathrm{n}(\%)$ & & & 0.19019 & & \\
\hline 0 & $238(39.14)$ & $261(41.10)$ & & Reference point & \\
\hline 1 & $201(33.06)$ & $198(31.18)$ & & $0.87(0.66-1.15)$ & 0.32757 \\
\hline 2 & $101(16.61)$ & $118(18.58)$ & & I.II (0.80-1.55) & 0.54278 \\
\hline 3 & $61(10.03)$ & $47(7.40)$ & & $0.71(0.46-1.09)$ & 0.11745 \\
\hline 4 & $5(0.82)$ & II (1.73) & & $2.36(0.79-7.09)$ & 0.12647 \\
\hline 5 & I $(0.16)$ & $0(0.00)$ & & 0 & 0.99958 \\
\hline 6 & I $(0.16)$ & $0(0.00)$ & & 0 & 0.99959 \\
\hline \multicolumn{6}{|l|}{ Cognitive profile } \\
\hline $\mathrm{MCl}, \mathrm{n}(\%)$ & 77 (I2.66) & I 30 (20.47) & 0.00022 & $1.72(1.25-2.38)$ & 0.00096 \\
\hline \multirow[t]{2}{*}{ Depression, n (\%) } & $(n=4 \mid 5)$ & $(n=382)$ & 0.85584 & $1.06(0.77-1.45)$ & 0.75044 \\
\hline & $208(49.88)$ & $189(49.48)$ & & & \\
\hline MMSE, mean \pm SD & $28.42 \pm 1.90$ & $27.58 \pm 3.12$ & $<0.00001$ & $1.12(1.05-1.19)$ & 0.00029 \\
\hline MoCA, mean $\pm S D$ & $27.60 \pm 2.72$ & $26.45 \pm 4.09$ & $<0.00001$ & $1.09(1.04-1.15)$ & 0.00092 \\
\hline $\mathrm{FAB}$, mean $\pm \mathrm{SD}$ & $16.82 \pm 1.57$ & $16.16 \pm 2.22$ & $<0.0000$ I & $1.19(1.08-1.31)$ & 0.00046 \\
\hline
\end{tabular}

Notes: In the multivariate logistic regression model, ORs were adjusted for covariates of years of education, ethnicity, socioeconomic status, and occupation. Abbreviations: FAB, Frontal Assessment Battery; MoCA, Montreal Cognitive Assessment; MMSE, Mini-Mental State Examination; SD, standard deviation; SES, socioeconomic status; $\mathrm{MCl}$, mild cognitive impairment; OR, odds ratio; $\mathrm{Cl}$, confidence interval.

health screening events. While the relationship between cardiovascular risk factors and cognitive impairment is complex, it has been found previously that the combined synergistic effects of multiple vascular risk factors may expedite the process of cognitive decline. ${ }^{32}$ This study found a prior history of stroke to be more correlated with cognitive dysfunction, which is consistent with previous findings..$^{36,37}$ High cholesterol in particular has been found to be significant 
among females, and has been examined previously. ${ }^{16,38}$ A recent study has found higher apolipoprotein e4-related risk of developing Alzheimer's dementia in females, and this also partly accounts for the female preponderance with MCI ${ }^{39}$ Vascular risk factors have been implicated in the etiology of dementia, ${ }^{40}$ hinting at a common neural basis and highlighting the importance of screening in this vulnerable cohort. However, longitudinal investigations are needed to elucidate causal relationships. In this population with comorbidities, visits to the doctor provide an ideal window of opportunity to screen for cognitive impairment and encourage a healthy lifestyle and management of medication. By managing all risk factors to address the multifactorial nature of cognitive decline, progression to dementia can be delayed.

MCI involves more substantial cognitive and memory decline than normal aging and represents a significant risk factor for development of dementia. ${ }^{41}$ Although there is no disease-modifying drug available specifically for dementia as yet, there are numerous benefits of recognizing cognitive impairments early. The lack of pharmacological therapies elevates the importance of non-pharmacological management, which also serves to prevent overmedicalization of patients, many of whom are already at high risk of polypharmacy. ${ }^{42}$ With more prompt detection, there is more time for these psychosocial measurements to be put in place, as opposed to late detection of moderate-to-severe dementia, when even hard-hitting pharmaceutical methods may only provide partial symptom control. Further, education is needed to optimize risk factors, including commencement and adherence with medications and lifestyle changes. Moreover, the importance of identifying MCI is set to increase with the eventual advent of trials involving newer preventative drugs, such as secretase inhibitors and immunotherapies. ${ }^{43}$ These key learning points have to be conveyed to the public in order to remove barriers and increase uptake of screening.

The profile of the screened cognitively intact population differs from that of the MCI group. Hence, if nationwide screening is applied on a voluntary basis, the population served would be people who are financially secure and at low risk of being missed by the health system. More vulnerable groups may be missed due to a lack of awareness or access, and so more aggressive methods are needed, such as a houseto-house approach or targeted community screening. This mismatch highlights the gap in awareness of dementia, and education should be targeted specifically to the group that is at a higher risk.
The sociodemographic characteristics of those older participants ( $\geq 61$ years) were male sex, few years of education, higher socioeconomic status, not gainfully employed, and lower-level occupation (Table 4). Higher proportions of older participants were found to have cardiovascular risk factors of diabetes mellitus, hypertension, high cholesterol, and heart disease. In terms of cognitive performance, there were higher proportions of persons with $\mathrm{MCI}$ in the older age groups, and their performance on the MMSE, MoCA, and FAB was noticeably poorer relative to that of their younger counterparts. Given that the Asian community-dwelling elderly 61 years and above constitute a high-risk group for developing MCI, public education efforts should actively target this older age group.

To our knowledge, this is the first study to describe the characteristics of an Asian population that takes up voluntary cognitive screening using a large cohort. We assessed both cognitive and clinical parameters using standardized and locally validated tools and protocols with trained staff, which would increase the sensitivity of detecting cognitive impairment. However, the limitations include large proportions of relatively highly educated (87.0\%), Chinese (91.2\%), and female $(67.0 \%)$ participants. Our population may therefore have a different set of characteristics when compared with the rest of Singapore as a whole, and our findings may not necessarily be generalizable. Due to the opportunistic cross-sectional design of this study, cognitive tests and demographic factors collated over the years are not uniform. Given the retrospective nature of this study, its results should be interpreted with caution, and causal inferences cannot be drawn. Further, there were ceiling effects of the cognitive tests used, which masked differences in cognitive function between the MCI and non-MCI groups. Future work could include more challenging cognitive tasks, using a more comprehensive and robust battery of neuropsychological assessments to elucidate these differences.

The unique design of this study has allowed insight into the awareness of cognitive impairment in the population and identified risk factors in the Asian population for MCI. Results from this study may provide preliminary evidence that the presence of a low level of education and vascular risk factors are general predisposing characteristics for MCI, with specific factors pertaining to sex and employment status. It is clear that targeted free screening with addition of informant report is the way forward. Public education on early recognition of MCI and optimizing risk factors is also crucial in staving off the debilitating consequences of cognitive impairment. 


\section{Acknowledgment}

This research was supported by the National Neuroscience Institute, Singapore, under the auspices of SingHealth.

\section{Author contributions}

$\mathrm{VH}$ and NHZ had full access to all of the data in the study and take responsibility for the integrity of the data and the accuracy of the data analysis, as well as the preparation, review, or approval of the manuscript. AN, LL, ES, and NK were responsible for the design and execution of the cognitive screening exercise over the 6-year study period, including logistic arrangements, cognitive evaluation, and consultation; collection, management, analysis, and interpretation of the data; preparation, review, or approval of the manuscript; and decision to submit the manuscript for publication. The sponsors were responsible for the design and execution of the cognitive screening exercise over the 6-year study period, including logistic arrangements, cognitive evaluation, and consultation. The sponsors were not responsible for the collection, management, analysis, or interpretation of the data, or the preparation, review, or approval of the manuscript, or decision to submit the manuscript for publication. All authors contributed toward data analysis, drafting and revising the paper and agree to be accountable for all aspects of the work.

\section{Disclosure}

This study was not funded by any grant or award. The authors report no conflicts of interest in this work.

\section{References}

1. World Health Organization. Dementia: a public health priority. Geneva, Switzerland: World Health Organization; 2012. Available from: http:// www.who.int/mental_health/publications/dementia_report_2012/en/. Accessed January 29, 2015.

2. Ferri CP, Prince M, Brayne C, et al; Alzheimer's Disease International. Global prevalence of dementia: a Delphi consensus study. Lancet. 2005;366:2112-2117.

3. Sahadevan S, Saw SM, Gao W, et al. Ethnic differences in Singapore's dementia prevalence: the stroke, Parkinson's disease, epilepsy and dementia in Singapore study. $J$ Am Geriatr Soc. 2008;56: 2061-2068.

4. Agüero-Torres H, von Strauss E, Viitanen M, Winblad B, Fratiglioni L. Institutionalisation in the elderly: the role of chronic diseases and dementia. Cross-sectional and longitudinal data from a population-based study. J Clin Epidemiol. 2001;54:795-801.

5. de Vugt ME, Verhey FR. The impact of early dementia diagnosis and intervention on informal caregivers. Prog Neurobiol. 2013;110:54-62.

6. Bischkopf J, Busse A, Angermeyer MC. Mild cognitive impairment a review of prevalence, incidence and outcome according to current approaches. Acta Psychiatr Scand. 2002;106:403-414.

7. Panza F, Capurso C, D'Introno A, et al. Mild cognitive impairment: dementia risk factor or high-risk state for progression to dementia? $J$ Am Geriatr Soc. 2008;56:1367-1369.
8. Richards M, Hardy R, Wadsworth ME. Does active leisure protect cognition? Evidence from a national birth cohort. Soc Sci Med. 2003;56: 785-792.

9. Patel BB, Holland NW. Mild cognitive impairment: hope for stability, plan for progression. Cleve Clin J Med. 2012;79:857-864.

10. Clark F, Jackson J, Carlson M, et al. Effectiveness of a lifestyle intervention in promoting the well-being of independently living older people: results of the Well Elderly 2 randomised controlled trial. J Epidemiol Community Health. 2012;66:782-790.

11. Jha A, Tabet N, Orrell M. To tell or not to tell - comparison of older patients' reaction to their diagnosis of dementia and depression. Int J Geriatr Psychiatry. 2001;16:879-885.

12. Alzheimer Europe. Value of knowing. Available from: http:/www. alzheimer-europe.org/Research/Value-of-Knowing. Accessed June 27, 2014.

13. Alzheimer's Disease International. Kyoto Declaration. Available from: http://www.alz.co.uk/adi/pdf/kyotodeclaration.pdf. Accessed June 27, 2014.

14. World Health Organization. WHO Mental Health Gap Action Programme (mhGAP). Available from: http://www.who.int/mental_health/ mhgap/en/. Accessed June 27, 2014.

15. Health Promotion Board, Singapore Government. Dementia. Available from: http://www.hpb.gov.sg/HOPPortal/dandc-article/11890?orginal Id=HPB053022. Accessed June 27, 2014.

16. Solfrizzi V, Panza F, Colacicco AM, et al; Italian Longitudinal Study on Aging Working Group. Vascular risk factors, incidence of MCI and rates of progression to dementia. Neurology. 2004;63:1882-1891.

17. Israeli-Korn SD, Masarwa M, Schechtman E, et al. Hypertension increases the probability of Alzheimer's disease and of mild cognitive impairment in an Arab community in northern Israel. Neuroepidemiology. 2010;34:99-105.

18. Su X, Shang L, Xu Q, et al. Prevalence and predictors of mild cognitive impairment in Xi' an: a community-based study among the elders. PLoS One. 2014;9:e83217.

19. Ritchie K, Ancelin ML, Beaino E, et al. Retrospective identification and characterisation of mild cognitive impairment from a prospective population cohort. Am J Geriatr Psychiatry. 2010;18:692-700.

20. Folstein MF, Folstein SE, McHugh PR. Mini-Mental State: a practical method for grading the cognitive state of patients for the clinician. J Psychiatr Res. 1975;12:189-198.

21. Dubois B, Slachevsky A, Litvan I, Pillon B. The FAB: a Frontal Assessment Battery at the bedside. Neurology. 2000;55:1621-1626.

22. Sheikh JI, Yesavage JA. Geriatric Depression Scale. Recent evidence and development of a shorter version. In: Brink TL, editor. Clinical Gerontology: A Guide to Assessment and Intervention. Philadelphia, PA, USA: The Haworth Press Inc; 1986.

23. Lam CK, Lim PP, Low BL, Ng LL, Chiam PC, Sahadevan S. Depression in dementia: a comparative validation study of four brief scales in the elderly Chinese. Int J Geriatr Psychiatry. 2004;19:422-428.

24. Crook TH 3rd, Feher EP, Larrabee GJ. Assessment of memory complaint in age-associated memory impairment: the MAC-Q. Int Psychogeriatr. 1992;4:165-176.

25. Lawton MP, Brody EM. Assessment of older people: self-maintaining and instrumental activities of daily living. Gerontologist. 1969;9:179-186.

26. Ng TP, Niti M, Chiam PC, Kua EH. Physical and cognitive domains of the Instrumental Activities of Daily Living: validation in a multiethnic population of Asian older adults. J Gerontol A Biol Sci Med Sci. 2006;61:726-735

27. Petersen RC, Smith GE, Waring SC, Ivnik RJ, Kokmen E, Tangelos EG. Aging, memory and mild cognitive impairment. Int Psychogeriatr. 1997;9 Suppl 1:65-69.

28. Ng TP, Niti M, Chiam PC, Kua EH. Ethnic and educational differences in cognitive test performance on MMSE in Asians. Am J Geriatr Psychiatry. 2007;15:130-139.

29. American Psychiatric Association. Diagnostic and Statistical Manual of Mental Disorders: Fifth Edition. Washington, DC, USA: American Psychiatric Association; 2013. 
30. Lawrence JM, Davidoff DA, Katt-Lloyd D, Connell A, Berlow YA, Savoie JA. Is large-scale community memory screening feasible? Experience from a regional memory-screening day. J Am Geriatr Soc. 2003;51:1072-1078.

31. Crews WD Jr, Harrison DW, Keiser AM, Kunze CM. The memory screening outreach program: Findings from a large communitybased sample of middle-aged and older adults. J Am Geriatr Soc. 2009;57:1697-1703.

32. Dregan A, Stewart R, Gulliford MC. Cardiovascular risk factors and cognitive decline in adults aged 50 and over: a population-based cohort study. Age Ageing. 2012;42:1-8.

33. Stern Y. Cognitive reserve in ageing and Alzheimer's disease. Lancet Neurol. 2012;11:1006-1012.

34. Farfel JM, Nitrini R, Suemoto CK, et al. Very low levels of education and cognitive reserve: a clinicopathologic study. Neurology. 2013;81:650-657.

35. Borson S, Scanlan JM, Watanabe J, Tu SP, Lessig M. Simplifying detection of cognitive impairment: comparison of the Mini-Cog and Mini-Mental State examination in a multiethnic sample. J Am Geriatr Soc. 2005;53:871-874.

36. Grau-Olivares M, Arboix A. Mild cognitive impairment in stroke patients with ischemic cerebral small-vessel disease: a forerunner of vascular dementia? Expert Rev Neurother. 2009;9:1201-1217.
37. Melkas S, Jokinen H, Hietanen M, et al. Poststroke cognitive impairment and dementia: prevalence, diagnosis, and treatment. Degener Neurol Neuromuscul Dis. 2014:21-27.

38. Yaffe K, Barrett-Connor E, Lin F, Grady D. Serum lipoprotein levels, statin use, and cognitive function in older women. Arch Neurol. 2002;59:368-384.

39. Altmann A, Tian L, Henderson VW, et al. Sex modifies the APOE-related risk of developing Alzheimer disease. Ann Neurol. 2014;75:563-573.

40. Kivipelto M, Ngandu T, Fratiglioni L, et al. Obesity and vascular risk factors at midlife and the risk of dementia and Alzheimer disease. Arch Neurol. 2005;62:1556-1560.

41. Davis HS, Rockwood K. Conceptualization of mild cognitive impairment: a review. Int J Geriatr Psychiatry. 2004;19:313-319.

42. Taipale H, Koponen M, Tanskanen A, Tolppanen AM, Tiihonen J, Hartikainen S. Antipsychotic polypharmacy among a nationwide sample of community-dwelling persons with Alzheimer's disease. J Alzheimers Dis. 2014;41:1223-1228.

43. Levey A, Lah J, Goldstein F, Steenland K, Bliwise D. Mild cognitive impairment: an opportunity to identify patients at high risk for progression to Alzheimer's disease. Clin Ther. 2006;28:991-1001.
Clinical Interventions in Aging

\section{Publish your work in this journal}

Clinical Interventions in Aging is an international, peer-reviewed journal focusing on evidence-based reports on the value or lack thereof of treatments intended to prevent or delay the onset of maladaptive correlates of aging in human beings. This journal is indexed on PubMed Central, MedLine,

\section{Dovepress}

CAS, Scopus and the Elsevier Bibliographic databases. The manuscript management system is completely online and includes a very quick and fair peer-review system, which is all easy to use. Visit http://www.dovepress. com/testimonials.php to read real quotes from published authors. 